OPEN ACCESS

Edited by:

Shucai Wang

Northeast Normal University, China

Reviewed by:

Sandeep Sharma,

Council of Scientific and Industrial Research (CSIR), India

Xianzhong Feng,

Northeast Institute of Geography and Agroecology (CAS), China

${ }^{*}$ Correspondence:

Gongwei Wang

gwwang@mail.hzau.edu.cn

Specialty section: This article was submitted to

Plant Physiology,

a section of the journal

Frontiers in Plant Science

Received: 06 August 2017 Accepted: 28 September 2017

Published: 13 October 2017

Citation:

Wang Q, Zhao H, Jiang J, Xu J, Xie W, Fu X, Liu C, He Y and Wang G (2017)

Genetic Architecture of Natural Variation in Rice Nonphotochemical Quenching Capacity Revealed by Genome-Wide Association Study. Front. Plant Sci. 8:1773. doi: 10.3389/fpls.2017.01773

\section{Genetic Architecture of Natural Variation in Rice Nonphotochemical Quenching Capacity Revealed by Genome-Wide Association Study}

\author{
Quanxiu Wang, Hu Zhao, Junpeng Jiang, Jiuyue Xu, Weibo Xie, Xiangkui Fu, Chang Liu, \\ Yuqing He and Gongwei Wang *
}

National Key Laboratory of Crop Genetic Improvement, National Center of Plant Gene Research (Wuhan), Huazhong Agricultural University, Wuhan, China

The photoprotective processes conferred by nonphotochemical quenching (NPQ) serve fundamental roles in maintaining plant fitness and sustainable yield. So far, few loci have been reported to be involved in natural variation of NPQ capacity in rice (Oryza sativa), and the extents of variation explored are very limited. Here we conducted a genome-wide association study (GWAS) for NPQ capacity using a diverse worldwide collection of 529 O. sativa accessions. A total of 33 significant association loci were identified. To check the validity of the GWAS signals, three F2 mapping populations with parents selected from the association panel were constructed and assayed. All QTLs detected in mapping populations could correspond to at least one GWAS signal, indicating the GWAS results were quite reliable. OsPSbS1 was repeatedly detected and explained more than $40 \%$ of the variation in the whole association population in two years, and demonstrated to be a common major QTL in all three mapping populations derived from inter-group crosses. We revealed 43 single nucleotide polymorphisms (SNPs) and 7 insertions and deletions (InDels) within a 6,997-bp DNA fragment of OsPsbS1, but found no non-synonymous SNPs or InDels in the coding region, indicating the PsbS1 protein sequence is highly conserved. Haplotypes with the 2,674-bp insertion in the promoter region exhibited significantly higher NPQ values and higher expression levels of OsPsbS1. The OsPsbS1 RNAi plants and CRISPR/Cas9 mutants exhibited drastically decreased NPQ values. OsPsbS1 had specific and high-level expression in green tissues of rice. However, we didn't find significant function for OsPsbS2, the other rice PsbS homologue. Manipulation of the significant loci or candidate genes identified may enhance photoprotection and improve photosynthesis and yield in rice.

Keywords: rice, nonphotochemical quenching, genome-wide association study, QTLs, OsPsbS1

\section{INTRODUCTION}

Although light is necessary for plants to drive photosynthesis, absorption of excess light by pigment molecules can cause severe photo-oxidative damage and inhibit photosynthesis. Most plants receive more sunlight than they can actually use for photochemistry reaction on a daily as well as seasonal basis (Murchie and Niyogi, 2011). In addition, light in plant canopies is very dynamic, and the levels 
of absorbed irradiance in leaves under field conditions can vary over several orders of magnitude in seconds (Külheim et al., 2002). Thus, to optimize photosynthesis and growth, plants have evolved a variety of photoprotective and photoacclimatory mechanisms that operate at different time scales (Li et al., 2009). For example, photoreceptors such as phototropin, neochrome, and cryptochrome can sense excess light and relay signals for chloroplast avoidance movement and gene expression responses. Excess excitation energy in the photosystem II (PSII) antenna complex can also be harmlessly dissipated as heat, which is observable as a process named nonphotochemical quenching of chlorophyll fluorescence (Müller et al., 2001; Li et al., 2002).

Nonphotochemical quenching (NPQ) can be divided into multiple components according to their relaxation kinetics in darkness following a period of illumination (Müller et al., 2001; Nilkens et al., 2010; Ruban, 2016). The major and most rapid component in plants is the $\mathrm{pH}$ - or energy-dependent component, $\mathrm{qE}$. The qE-type of NPQ or feedback de-excitation, is a major photoprotective strategy that operates on a timescale of seconds to minutes and involves a regulated thermal dissipation of excess absorbed light energy. The rapid induction and relaxation of $\mathrm{qE}$ are required to cope with frequent, rapid and irregular changes in the natural light environment (Külheim et al., 2002). A current model of $\mathrm{qE}$ in plants is as follows. When light absorption exceeds the capacity for light utilization in assimilatory reactions, a decrease in the proton conductance of the chloroplast ATPase results in a rapid decrease in thylakoid lumen $\mathrm{pH}$. The low thylakoid lumen $\mathrm{pH}$ induces de-epoxidation of violaxanthin to antheraxanthin and zeaxanthin via the xanthophyll cycle and protonation of a photosystem II protein, PsbS. Together, binding of protons and xanthophylls (zeaxanthin and lutein) to specific sites in the PSII antenna causes a conformational change that switches into a quenched state (Müller et al., 2001; Bianchi et al., 2010; Ruban, 2016).

Natural variation in NPQ capacity has been reported in different plant species. Jung and Niyogi (2009) surveyed the genetic basis for natural variation of NPQ among Arabidopsis accessions. In spite of significant differences in NPQ, previously identified NPQ factors such as xanthophyll cycle de-epoxidation state and the amount of the PsbS protein were indistinguishable between the high and the low NPQ accessions, suggesting that the differences in NPQ variation in Arabidopsis may be controlled by new genes. Using a F2 mapping population, two high NPQ QTLs were identified and one was validated by the phenotype of near isogenic lines (Jung and Niyogi, 2009). In rice, Kasajima et al. (2011) identified significant differences in $\mathrm{qE}$ capacity between indica and japonica cultivars. Using backcrossed inbred lines derived from an inter-subspecific cross, two QTLs on chromosome 1 were identified for the magnitude of NPQ, with one harboring the OsPsbS1 gene. Comparison of the public available genomic sequence around $O s P s b S 1$ revealed that a 2.7$\mathrm{kb}$ region upstream of the translation initiation site of OsPsbS1 was lost in 9311 (indica) compared with Nipponbare (japonica). Existence of this deletion was also analyzed by PCR markers in several japonica and indica cultivars, and this deletion was found to be correlated with NPQ capacity (Kasajima et al., 2011). Nuruzzaman et al. (2014) further demonstrated the 2.7-kb insertion fragment in Nipponbare contains a Mutator-likeelement (MULE). However, the nucleotide diversity, haplotype effects, and expression patterns of OsPsbS1 haven't been characterized in detail.

As the staple food for more than half of the world's population, rice is normally cultivated under high natural illumination in summer season, and light may be frequently in excess of that required for $\mathrm{CO}_{2}$ assimilation. The ability of $\mathrm{qE}$ to safely dissipate excess excitation energy is thus very important for plant fitness and sustainable yield in rice. Although OsPsbS1 has been identified (Kasajima et al., 2011), the extent of variations in NPQ capacity has not been fully explored due to the very limited number of rice accessions used previously. Compared with QTL linkage mapping approach, genome-wide association study (GWAS) can greatly increase the range of natural variation and the number of significant loci, especially for complex traits (Huang et al., 2010; Zhao et al., 2011). In this study, we conducted a GWAS for NPQ capacity using a diverse worldwide collection of 529 O. sativa accessions. Thirty-three significant association regions were identified, and three F2 mapping populations with parents selected from the association panel were tested for validation of significant GWAS signals. OsPsbS1 was found to be a major determinant for natural variation of NPQ capacity. To our knowledge, this study provides the most comprehensive investigation into the genetic architecture of natural variation of NPQ capacity in rice to date. The nucleotide diversity, haplotype effects, and expression patterns of OsPsbS1 were further characterized. The function of OsPsbS1 was verified by RNA interference and CRISPR/Cas9 knockout mutants. The implications of the results were discussed.

\section{MATERIALS AND METHODS}

\section{Plant Materials for Association Analysis}

The association panel consisted of a diverse collection of 529 O. sativa accessions including both landraces and elite varieties. The details about the accessions, including accession name, country of origin, longitude and latitude origin, and subpopulation identity, have been reported previously (Wang et al., 2015; Xie et al., 2015) and are available at the RiceVarMap (http://ricevarmap.ncpgr.cn).

\section{Genome-Wide Association Analyses}

The sequencing, SNP identification, and imputation of the association panel have been reported in previous studies (Wang et al., 2015; Xie et al., 2015). The SNPs of the 529 O. sativa accessions are available at the RiceVarMap (http://ricevarmap.ncpgr.cn). There were 2,767,191, 1,857,866, $1,925,362$, and $3,916,415$ SNPs (minor allele frequency $\geq 0.05$; the number of accessions with minor alleles $\geq 6$ ) used in GWAS for groups of Indica, Japonica, Aus, and the whole panel, respectively. We performed GWAS using the linear mixed model (LMM) and the simple linear regression model (LR) provided by FaSTLMM program (Lippert et al., 2011). The population structure of $\mathrm{Q}$ matrix and kinship ( $\mathrm{K}$ matrix) was taken into account as cofactor when performing association mapping using the LMM method. Using a method described by Li et al. (2012), the 
effective numbers of independent SNPs and suggestive thresholds were calculated. The effective numbers of independent SNPs were 571,843, 245,348, 235,880, and 757,578 for groups Indica, Japonica, Aus, and the whole panel, respectively. The suggestive $P$ values set as thresholds to identify significant association signals by LMM were $1.75 \times 10^{-6}, 4.08 \times 10^{-6}, 4.24 \times 10^{-6}$, and 1.32 $\times 10^{-6}$ for groups Indica, Japonica, Aus, and the whole panel, respectively. To obtain independent association signals, multiple SNPs exceeding the threshold in a $5 \mathrm{Mb}$ region were clustered by $r^{2}$ of LD $\geq 0.25$ and SNPs with the minimum $P$-value in a cluster were considered as lead SNPs.

\section{Field Experiments and Evaluation of NPQ Values in the Association Panel}

Field trials were conducted in two years. The rice seeds were sown in the Experimental Station of Huazhong Agricultural University, Wuhan, China, in mid-May of 2012 and 2013. Seedlings about 25 days old were transplanted to the field. Each plot consisted of 4 rows with 10 plants each. The planting density was $16.5 \mathrm{~cm}$ between plants in a row, and the rows were $26 \mathrm{~cm}$ apart. Field management, including irrigation, fertilizer application and pest control, followed essentially the normal agricultural practice.

Three plants in the middle from the third row of each accession were subject to investigation for NPQ values before heading stage. Flag leaves were excised and floated on ionexchanged water containing $0.01 \%$ Triton $\mathrm{X}-100$ or $0.1 \%$ agar in centrifuge tubes. Leaves were dark-acclimated for at least 2 h. Chlorophyll fluorescence was measured with the PAM-2500 portable chlorophyll fluorometer (WALZ, Germany). Actinic lights were supplemented at a PPFD of $1,000 \mu \mathrm{mol} \mathrm{m} \mathrm{m}^{-2} \mathrm{~s}^{-1}$ for $5 \mathrm{~min}$ before measurements of $\mathrm{Fm}^{\prime}$ to calculate parameter NPQ. The Stern-Volmer type nonphotochemical fluorescence quenching (Bilger and Björkman, 1990) was calculated by the following equation: $\mathrm{NPQ}=\mathrm{Fm} / \mathrm{Fm}^{\prime}-1$.

\section{QTL Analysis}

Three F2 populations were constructed for QTL mapping, with parents selected from the association panel. All of them were derived from inter-group crosses, an IndI by TrJ cross (Sadu-cho/Dourado Precoce), an IndI by TeJ cross (Zegu/Weiguo), and an Aus by TeJ cross (Dular/Akitakomachi). For each F2 population, 144 individuals were investigated for genotypic and phenotypic assay. Genotypic assay was performed using SSR markers, and measurement of NPQ values was conducted with the same method as described above. QTL analysis was conducted using composite interval mapping in QTL Cartographer (http://statgen.ncsu.edu/qtlcart/ cartographer.html). The SSR markers used in the QTL analysis are presented in Table S4.

\section{Nucleotide Diversity Analysis}

The genomic DNA extraction and sequencing were carried out according to the methods described by Du et al. (2011). The DNA sequences were assembled using SeqMan software and all polymorphisms were confirmed visually to ensure the accuracy. Sequences were inspected initially using Sequencher V5.4 program and edited manually using the SeqMan software. The genomic sequences were aligned by MAGE5. Nucleotide diversity and Tajima's $D$ statistics were calculated using the DnaSP 5.0 program.

\section{Quantitative RT-PCR Analysis}

Total RNA was isolated from rice leaves using TRIzol Reagent (TransGen) according to the manufacturer's instructions. Approximately $3 \mu \mathrm{g}$ of RNA sample was subjected to RNase-free DNaseI (Invitrogen) treatment and reverse transcribed using M-MLV Reverse Transcriptase (Invitrogen) with Oligo(dT)15. Quantitative RT-PCR was performed in a ViiA 7 Real-Time PCR system (Applied Biosystems) using FastStart Universal SYBR Green Master (Rox) superMIX (Roche). Ubiquitin was used as a reference gene in the qRT-PCR experiments. The measurements were obtained using the relative quantification method. The significant difference was analyzed statistically by student's $t$ test or one-way analysis of variance (ANOVA). Primer pairs for qRT-PCR analysis are listed in Table S3.

\section{Vector Construction for RNAi Knockdown and CRISPR/Cas9 Knockout}

To generate the RNAi constructs, the 425-bp fragment from the exon of OsPsbS1 was amplified by PCR using specific primers (Table S3), and cloned into the KpnI-BamHI and SpeI-SacI restriction sites of RNAi construct ds 1301.

The CRISPR/Cas9 vector system for multiplex targeting of gene sites in monocot plants was kindly provided by Professor Yaoguang Liu (South China Agriculture University). In order to increase the efficiency of the targeting, we designed two target special sequences for each gene in exon regions. Briefly, the target site sequences were cloned into the sgRNA expression cassette which contains rice promoter, and then cloned into the BsaI restriction sites of vector $\mathrm{pYLCRISPR/Cas9-MH.} \mathrm{The} \mathrm{primers}$ used in constructing the sgRNA vectors for OsPsbS1 and OsPsbS2 are listed in Table S3.

The RNAi and CRISPR/Cas9 constructs were independently introduced into Agrobacterium tumefaciens strain EHA105, and then transformed into Zhonghua 11.

\section{Histochemical Staining of GUS Activity}

Histochemical staining of GUS activity in rice tissues was conducted essentially as described previously. Various tissues of transgenic-positive transformants (root, leaf, sheath, panicle, stem and mature seed) were incubated in GUS staining solution (50 mM sodium phosphate at $\mathrm{pH} 7.0,10 \mathrm{mM} \mathrm{Na}_{2}$-EDTA, 0.1\% Triton X-100, $1 \mathrm{mg} / \mathrm{ml}$ X-Gluc, $100 \mu \mathrm{g} / \mathrm{ml}$ chloramphenicol, 1 $\mathrm{mM}$ potassium ferricyanide, $1 \mathrm{mM}$ potassium ferrocyanide, and $20 \%$ methanol) at $37^{\circ} \mathrm{C}$ for $2-10 \mathrm{~h}$ after 15 -min vacuum filtration. After GUS staining, the samples were incubated in $70 \%$ ethanol to remove chlorophyll and photographs were taken under a dissecting microscope (Leica MZFLIII).

\section{RESULTS}

\section{Phenotypic Variation of NPQ in the Association Panel}

As described by us previously (Wang et al., 2015; Xie et al., 2015), the whole association panel exhibited a distinct population structure, and was classified into 98 indica I (IndI), 105 
indica II (IndII), 92 indica intermediate, 91 temperate japonica $(\mathrm{TeJ}), 44$ tropical japonica (TrJ), 21 japonica intermediate, 46 Aus, and 14 VI (an intermediate group). The basic information of 529 accessions is available at the RiceVarMap (http://ricevarmap.ncpgr.cn).

The mean values and standard deviation of NPQ in different groups and all accessions in 2012 and 2013 (designated as NPQ_12 and NPQ_13, respectively) are presented in Table 1. A significant correlation was observed between NPQ_12 and NPQ_13 in the whole association population $(r=0.64)$. Large phenotypic variation was mainly detected between different subspecies in both years. Comparisons of the trait values between indica (consisting of IndI, IndII and indica intermediate) and japonica (consisting of $\mathrm{TeJ}, \mathrm{TrJ}$ and japonica intermediate) revealed that japonica generally had significantly higher values in NPQ_12 $\left(P=2.79 \times 10^{-47}\right)$ and NPQ_13 $\left(P=2.47 \times 10^{-59}\right)$. Aus exhibited similar magnitude of NPQ values to indica $(P>$ $0.05)$ in both two years, while much lower values than that in japonica $\left(P=7.68 \times 10^{-11}\right.$ for NPQ_12 and $P=1.29 \times 10^{-17}$ for NPQ_13). However, no statistically significant differences in NPQ values were observed between IndI and IndII or between $T e J$ and $T r J$, with the only exception occurred between IndI and IndII in NPQ_13 $(P=0.00028)$.

\section{Identification of Significant Loci for NPQ Values through GWAS}

Genome-wide association analyses were performed separately in the whole population and in the indica, japonica, and aus subpopulations for each year. A total of 33 significant association regions were identified at linear mixed models (LMM) (lead SNPs less than or around $300 \mathrm{~kb}$ were considered as caused by one common gene and counted as only one association region) for NPQ values. The details about these significant association signals are listed in Table 2. The quantilequantile plots and Manhattan plots of LMM for NPQ_13 in the whole population are illustrated in Figure $\mathbf{1}$ as an example.

GWAS results over the two years were compared. Among them, two regions were commonly detected in both NPQ_12 and NPQ_13. Significant genetic heterogeneity was observed when comparing GWAS results performed in the different

TABLE 1 | The means and standard deviation of NPQ values in different groups and the whole panel in 2012 and 2013.

\begin{tabular}{llllll}
\hline \multirow{2}{*}{ Group } & \multicolumn{2}{c}{ NPQ_12 } & & \multicolumn{2}{c}{ NPQ_13 } \\
\cline { 2 - 3 } & Means & SD & & Means & SD \\
\hline Indl & 2.371 & 0.209 & & 2.450 & 0.247 \\
Indll & 2.366 & 0.175 & & 2.564 & 0.165 \\
TeJ & 2.740 & 0.230 & & 2.941 & 0.208 \\
TrJ & 2.721 & 0.279 & & 2.980 & 0.296 \\
Aus & 2.421 & 0.213 & & 2.567 & 0.206 \\
Ind_All & 2.363 & 0.191 & & 2.522 & 0.211 \\
Jap_All & 2.716 & 0.256 & & 2.946 & 0.245 \\
All accessions & 2.487 & 0.273 & & 2.665 & 0.295
\end{tabular}

groups and in the whole population. Only three significant association regions were detected in at least two different groups (Table 2).

To search for candidate genes, the significant GWAS signals were compared with the positions of known or putative genes involved in NPQ regulation. We found that OsPsbS1 corresponds to one significant association region that was repeatedly detected and explained more than $40 \%$ of the variation in the whole population in both 2012 and 2013 (Table 2). However, no other previously identified NPQ key factors, such as genes related to xanthophyll cycle and lutein synthesis (Niyogi et al., 1998, 2001; Ruban, 2016), were found to be close to the detected significant association signals. It suggests the NPQ variation in rice may be controlled by a lot of new genes. Close to the GWAS peak signals, we found some candidate genes encoding light-induced protein 1-like, thylakoid lumenal protein, photosystem II (PSII) $10 \mathrm{kDa}$ polypeptide, ATPase, ATP synthase gamma chain, and plastocyanin-like domain containing protein (Table 2). These candidate genes might be involved in processes of electron transport, buildup of proton gradient, PSII light harvesting antenna structure and light harvesting complex II (LHCII) rearrangements that trigger and regulate NPQ scenario (Ruban, 2016).

\section{Validation of GWAS Signals with QTL Mapping}

To investigate whether GWAS significant signals could be detected in bi-parental QTL mapping, we constructed three F2 mapping populations derived from inter-group crosses, with parents selected from the association panel. In the F2 population of IndI by TrJ (Sadu-cho/Dourado Precoce), one major QTL qSD1.1, explaining $29.4 \%$ of the phenotypic variation, located in the interval between RM212 and RM6504 on chromosome 1, was identified. In addition, a QTL qSD8.1, located in the interval between RM556 and RM80 on chromosome 8, also showed significant effects on NPQ values. The detailed information about these significant QTLs is presented in Table 3.

In the IndI by $\mathrm{TeJ}$ (Zegu/Weiguo) F2 population, similarly, one major QTL qZW1.1, explaining 33.6\% of the phenotypic variation, located in the interval between RM8278 and RM315 on chromosome 1, was detected. Furthermore, a QTL qZW7.1, located in the interval between RM7571 and RM13.40 on chromosome 7, exhibited significant effects (Table 3).

In the F2 population of Aus by TeJ (Dular/Akitakomachi), one major QTL qDA1.1, explaining 37.0\% of the phenotypic variation, located in the interval between RM212 and RM315 on chromosome 1, was also identified. Additionally, two QTLs qDA7.1 (located in the interval between RM6344 and RM420 on chromosome 7) and qDA9.1 (located in the interval between RM5657 and RM434 on chromosome 9), showed significant effects on NPQ values (Table 3).

After comparing the regions of these QTLs with GWAS signals, we found that all the major QTL detected on chromosome 1 corresponds to OsPsbS1, and other significant QTLs also co-localize with at least one GWAS signal (Table 3), indicating the GWAS results were quite reliable. 
TABLE 2 | The details about the significant GWAS signals for NPQ values and candidate genes.

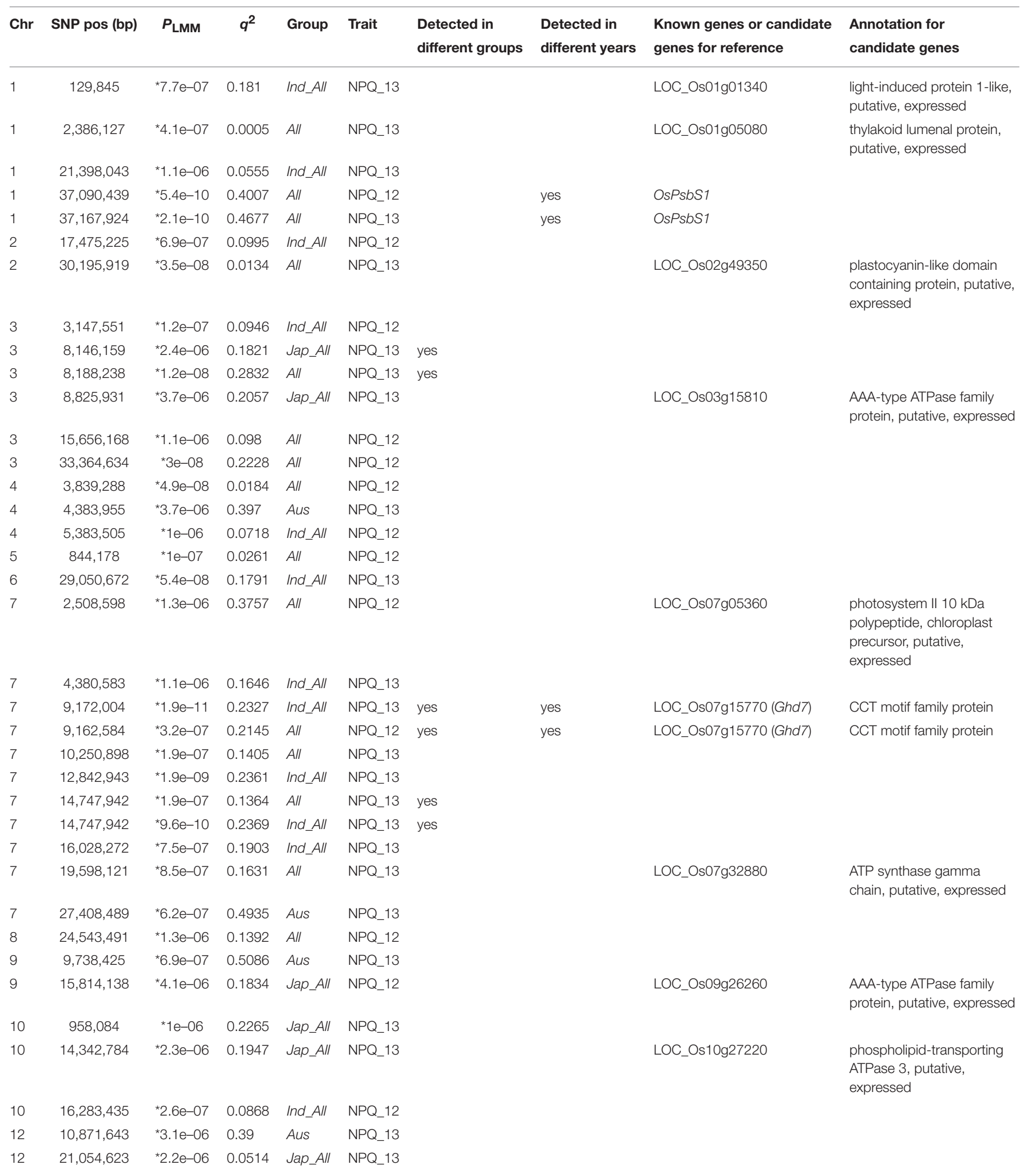

Chr, chromosome; SNP PoS, SNP position on rice genome assembly MSU version v6.1; $P_{L M M}, P$ value from $L M M$; $q^{2}$, variance explained by the $S N P$ effect; *indicates $P$ value from LMM was lower than the significance thresholds. 
Trait: NPQ_13 Pop: All
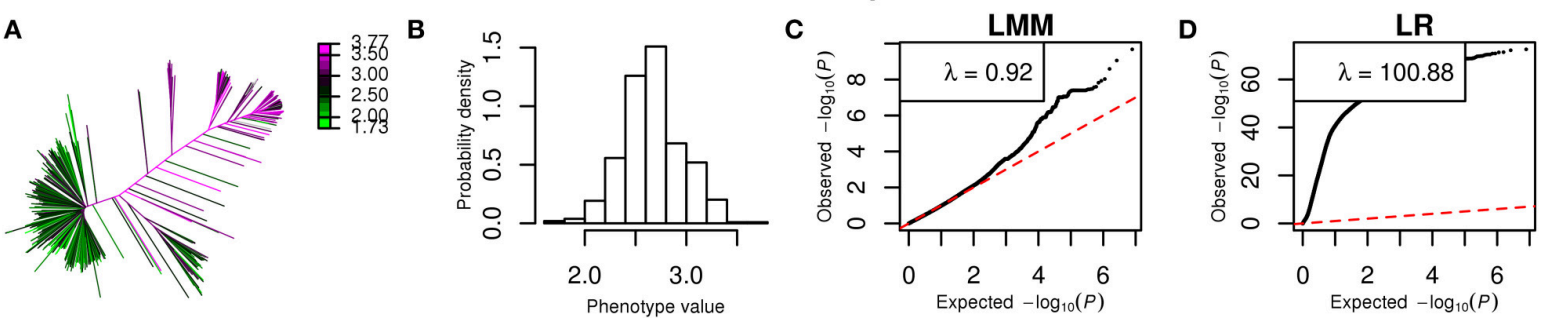

E

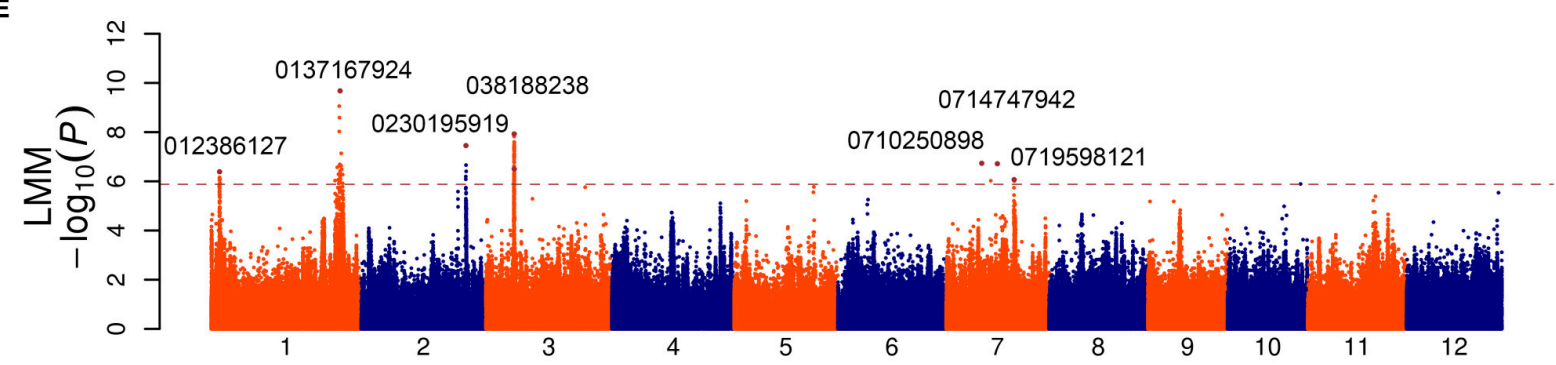

$\mathbf{F}$

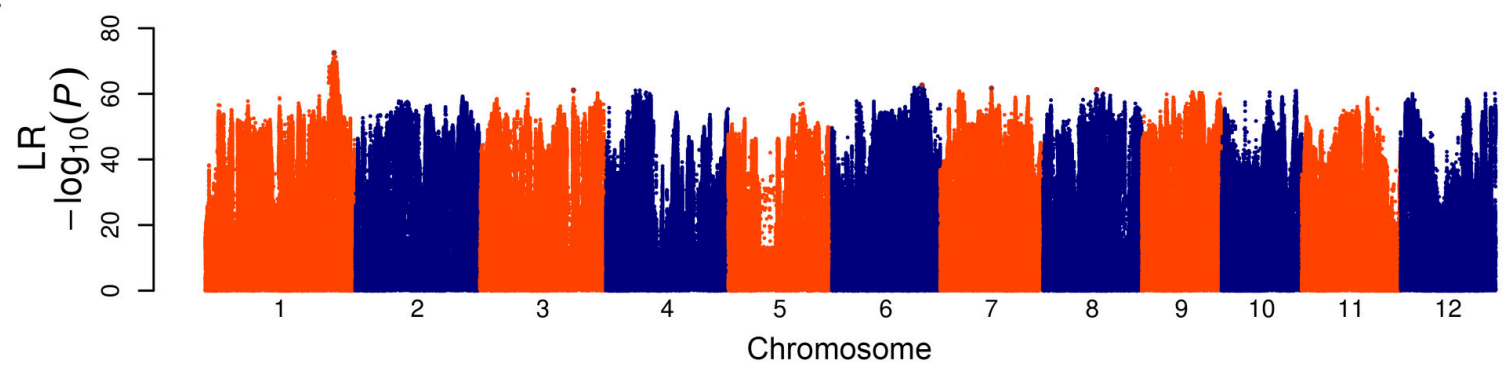

FIGURE 1 | GWAS of NPQ values in 2013 for the whole association population. (A,B) The heatmap (A) and histogram (B) distribution of NPQ values in 2013 in 529 accessions. (C,D) Q-Q plot of the expected null distribution and the observed $P$-value using the linear mixed model (LMM) (C) and the simple linear regression (LR) model (D). (E,F) Genome-wide $P$-values for the LMM (E) and simple LR model (F). The horizontal dashed line indicates the significance thresholds set as $P=1.32 \times$ $10^{-6}$ for the whole population by LMM. The SNP positions of representative peak signals in LMM were denoted.

TABLE 3 | Summary of significant QTLs for NPQ values identified in three F2 populations of this study.

\begin{tabular}{|c|c|c|c|c|c|c|c|c|}
\hline Cross of $F_{2}$ population & QTL & Chr & Interval & LOD & $\operatorname{Add}^{a}$ & Domb & $\operatorname{Var}(\%)^{\mathrm{c}}$ & $\begin{array}{l}\text { Corresponding lead SNP position } \\
\text { in GWAS or known genes }\end{array}$ \\
\hline Sadu-cho/Dourado Precoce $\left(I n d l{ }^{*} \operatorname{Tr} J\right)$ & qSD1.1 & 1 & RM212-RM6504 & 10.1 & -0.1724 & 0.0279 & 29.4 & OsPsbs1 \\
\hline \multirow[t]{2}{*}{ Zegu/Weiguo (Indl * TeJ) } & qZW1.1 & 1 & RM8278-RM315 & 12.6 & -0.1793 & 0.0253 & 33.6 & OsPsbs1 \\
\hline & qZW7.1 & 7 & RM7571-RM13.40 & 2.7 & -0.1369 & 0.0371 & 11.1 & $12,842,943$ \\
\hline \multirow[t]{2}{*}{ Dular/Akitakomachi (Aus * TeJ) } & qDA1.1 & 1 & RM212-RM315 & 13.7 & -0.1857 & 0.0212 & 37.0 & OsPsbs1 \\
\hline & qDA9.1 & 9 & RM5657-RM434 & 3.4 & -0.0764 & 0.0396 & 9.1 & $15,814,138$ \\
\hline
\end{tabular}

${ }^{a}$ Additive effects. Positive values indicate that the allele from Sadu-cho or Zegu or Dular can increase the phenotypic value, whereas negative values indicate the allele from the counterpart parent Dourado Precoce or Weiguo or Akitakomachi can increase the phenotypic value.

${ }^{b}$ Dominance effect. Positive values imply that the heterozygotes had higher phenotypic value than the means of the two homozygotes, whereas negative values imply that the heterozygotes had lower phenotypic value than the means of the two homozygotes.

${ }^{c}$ Variance explained.

\section{Characterization of OsPsbS1, a Major Locus for Natural Variation of NPQ Capacity in Rice}

To characterize the nucleotide diversity of OsPsbS1, the whole genomic DNA sequences around OsPsbS1 from 480 accessions in the association panel were sequenced and analyzed. In total, 43 SNPs and 7 insertions and deletions (InDels) were detected in the aligned 6,997 basepairs, which include 4,022bp promoter and 2975-bp coding regions (Table 4). Varied DNA polymorphisms were observed in different regions of the OsPsbS1 genome. We found no nucleotide polymorphic sites in exon-1, intron-1, and exon-2, and only one synonymous 
TABLE 4 | Summary of DNA polymorphic sites of OsPsbS1 genome.

\begin{tabular}{|c|c|c|c|c|c|c|c|c|c|}
\hline Parameter & Promoter & Gene region & 5'UTR & Exon-1 & Intron-1 & Exon-2 & Intron-2 & Exon-3 & $3^{\prime}$ UTR \\
\hline Length, bp & 4,022 & 2,975 & 331 & 198 & 116 & 117 & 1,379 & 492 & 342 \\
\hline SNP sites & 15 & 28 & 2 & 0 & 0 & 0 & 18 & 1 & 7 \\
\hline Indels & 2 & 5 & 0 & 0 & 0 & 0 & 4 & 0 & 1 \\
\hline \multicolumn{10}{|c|}{ WHOLE POPULATION } \\
\hline$\pi$ & 0.00224 & 0.00306 & 0.00283 & 0 & 0 & 0 & 0.00444 & 0.00024 & 0.00581 \\
\hline$\theta$ & 0.00165 & 0.00145 & 0.00090 & 0 & 0 & 0 & 0.00205 & 0.00030 & 0.00318 \\
\hline Tajima's D & 0.82255 & $2.89836^{\star \star}$ & $2.62209^{*}$ & - & - & - & $2.86141^{\star \star}$ & -0.18476 & 1.58889 \\
\hline \multicolumn{10}{|c|}{ japonica GROUP } \\
\hline$\pi$ & 0.00045 & 0.00033 & 0.00031 & 0 & 0 & 0 & 0.00050 & 0 & 0.00055 \\
\hline$\theta$ & 0.00146 & 0.00097 & 0.00108 & 0 & 0 & 0 & 0.00143 & 0 & 0.00164 \\
\hline Tajima's D & -1.71154 & -1.75267 & -1.01483 & - & - & - & -1.60050 & - & -1.11096 \\
\hline \multicolumn{10}{|c|}{ indica GROUP } \\
\hline$\pi$ & 0.00058 & 0.00131 & 0.00014 & 0 & 0 & 0 & 0.00242 & 0 & 0.00149 \\
\hline$\theta$ & 0.00109 & 0.00137 & 0.00098 & 0 & 0 & 0 & 0.00212 & 0 & 0.00339 \\
\hline Tajima's D & -1.02660 & -0.13007 & -1.12832 & - & - & - & 0.35855 & - & -0.74251 \\
\hline
\end{tabular}

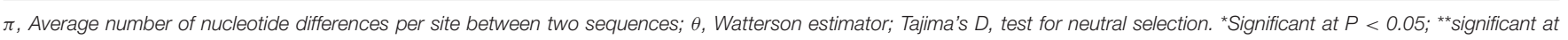
$P<0.01$.

SNP in exon-3, indicating the structure and function of PsbS protein are highly conserved in rice. In the whole germplasm population, the pairwise nucleotide diversity parameter $(\pi)$ and the level of the Watterson estimator $(\theta)$ in intron- 2 and $3^{\prime}$ UTR regions were much higher than that in the exons, promoter, and 5'UTR regions. Tajima's $D$ values reached a significant positive level in the OsPsbS1 gene region (Table 4). Considering the strong population stratification, we also tested these parameters within the two subpopulations (indica and japonica group). The sequence diversities of indica in intron2 and $3^{\prime}$ UTR regions were also much higher than that in the exons, promoter, and 5'UTR regions. Compared with indica, lower levels of nucleotide diversity were observed in japonica. The Tajima's $D$ values showed negative but not statistically significant values in both indica and japonica group (Table 4).

The sequencing results showed that the vast majority of japonica varieties has the 2,674-bp insertion fragment in the promoter region of OsPsbS1, compared with most indica and aus varieties. We further analyzed the effects of haplotypes based on 15 SNPs and 2 indels in the promoter region. For the strictness of statistical analysis, we only focused on those haplotypes shared by at least five accessions. The 480 accessions were accordingly divided into 7 haplotypes, among which hap1-3 contained the 2674-bp insertion, whereas hap4-7 didn't (Figure 2A). It was found that haplotypes with the 2674-bp insertion exhibited significantly higher values in NPQ_12 (Figure 2B) and NPQ_13 (Figure 2C) than other haplotypes without the insertion. We also compared the expression levels of OsPsbS1 in flag leaves of 49 randomly chosen accessions with the two major haplotypes, hap1 and hap4. Real-time PCR results showed that the expression level of OsPsbS1 in hap1 was much higher than that in hap4 (Figure 2D). The results suggest that variation of the OsPsbS1 expression levels might be the main cause of natural variation of NPQ capacity in the whole association panel.

To verify the function of OsPsbS1 in vivo, we first utilized RNA interference to silence the expression of OsPsbS1. The 425bp fragment from the exon of OsPsbS1 was cloned into RNAi constructs ds1301, which were then transformed into Zhonghua 11, a japonica variety. Further real-time PCR analysis showed that the transcription of $O s P s b S 1$ was drastically reduced in all transformants with much lower NPQ values (Figures 3A,B).

In the sequenced rice genome, OsPsbS2, another chlorophyll A-B binding protein gene, is found to be most similar to OsPsbS1 with a nucleotide identity of $91 \%$. To distinguish the function of these two homologous genes, we then used the CRISPR-Cas9 system with constructs containing Cas9 and sgRNA and targeting the single gene OsPsbS1 or OsPsbS2 and both of the two genes. The constructs were transformed into a japonica variety Zhonghua 11 . Two target special sequences were designed for each gene. The potential mutations were detected by sequencing the PCR amplification products containing the two targets regions. Indels mutations were confirmed in the target regions. Compared with the wild-type plants, the OsPsbS1 mutants and the double mutants exhibited much lower NPQ values (Figure 3C). However, no significant differences were found in NPQ values between the OsPsbS2 mutants and the wild-type plants (Figure 3C).

To get an overview of the expression profile of OsPsbS1, the CREP database (http://crep.ncpgr.cn/crep-cgi/home.pl), a website containing the dynamic gene expression atlas of indica rice, was searched (Wang et al., 2010). It was found that OsPsbS1 exhibited high-level expression in leaf but low-level expression in root and stem in Minghui 63 and Zhenshan 97, two indica cultivars (Figure 4A). In addition, we also investigated the relative expression level of OsPsbS1 in 02428, a japonica variety, by quantitative RT-PCR. Similarly, it was 


\section{A}

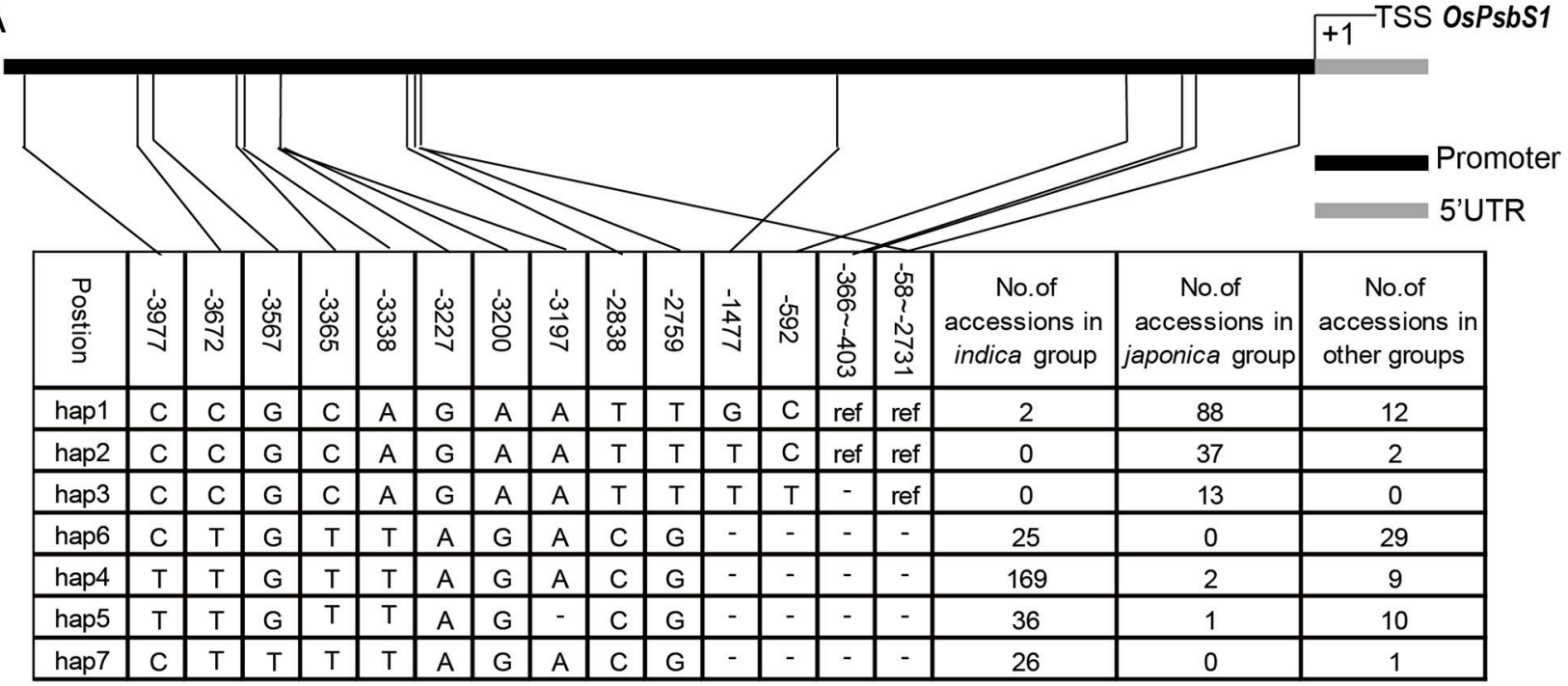

B

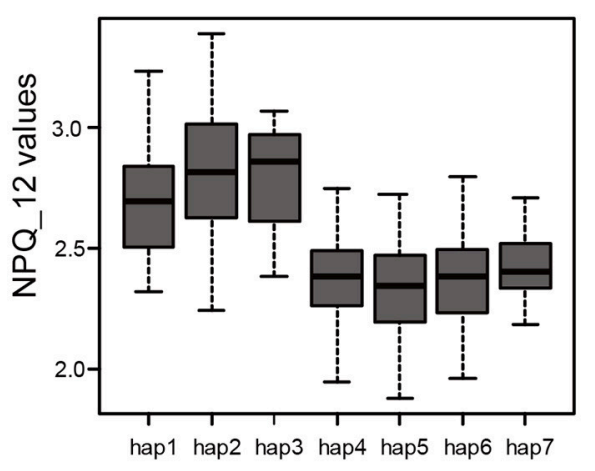

C

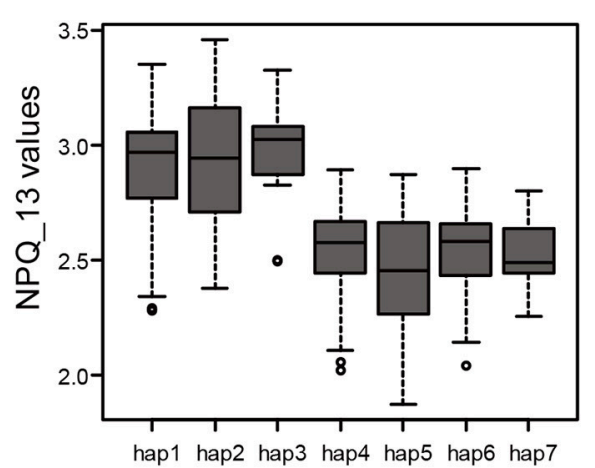

D

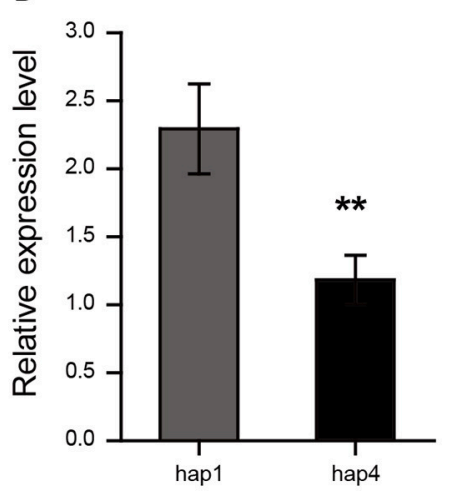

FIGURE 2 | Haplotype analysis of the OsPsbS1 promoter region in the 480 accessions. (A) Seven haplotypes (hap1-hap7) were detected. The 4,022-bp promoter region is shown in graphics on the top. The position of every SNP is shown in the first row. The number of accessions in each haplotype is shown in the right columns. Ref, sequence identical to Nipponbare; -, sequence deleted. (B) The distribution of NPQ_12 values in the seven haplotypes. (C) The distribution of NPQ_13 values in the seven haplotypes. (D) The transcript levels of OsPsbS1 in flag leaves of hap1 and hap4 accessions. ${ }^{\star *}$ The differences in expression levels between hap1 and hap4 are significant at $P<0.01$.

highly expressed in green tissues including leaf and leaf sheath (Figure 4B), indicating OsPsbS1 was a green tissue-specific expressed gene. To complement the results at the mRNA level, a 3141-bp promoter region of OsPsbS1 was ligated in frame with the $\beta$-glucuronidase (GUS) reporter gene, which was then introduced into Zhonghua 11, a japonica variety. GUS staining of the $P_{O s P s b S 1}:: G U S$ transgenic plants showed that OsPsbS1 had specific and high-level expression in mature leaf (Figure 4E), leaf sheath (Figure 4F), and young glume (Figure 4G), which was in accordance with its high expression in the green tissues of 02428 . No staining signals was observed in root (Figure 4C), stem (Figure 4D), and mature seed (Figure 4H). We further compared the expression levels of OsPsbS1 in various tissues before heading stage between two varieties with different genotypes in the OsPsbS1 promoter region by quantitative RT-PCR (Figure 4I). Zhonghua 11 contains the 2,674-bp insertion fragment in the promoter region, whereas Zhenshan 97 doesn't. The results showed that the expression level of OsPsbS1 in Zhonghua 11 was much higher than that in Zhenshan 97 in green tissues such as leaves and shealth (Figure 4I). However, OsPsbS1 exhibited very low-level expression in root and stem in both Zhonghua 11 and Zhenshan 97 (Figure 4I). In conclusion, OsPsbS1 had specific and high-level expression in green tissues of rice, and genotypes with the 2674-bp insertion fragment in the promoter region tend to exhibit much higher level expression.

\section{Analysis of One Candidate Gene}

A candidate gene encoding light-induced protein 1-like (LOC_Os01g01340) was located in one significant association region and close to the representative GWAS peak signal (around 129,845 on chromosome 1) for NPQ_13 in indica group (Table 2). Using resequencing data, the haplotypes of LOC_Os01g01340 were built based on SNPs and InDels detected in the promoter region (around $1 \mathrm{~kb}$ upstream of transcription 


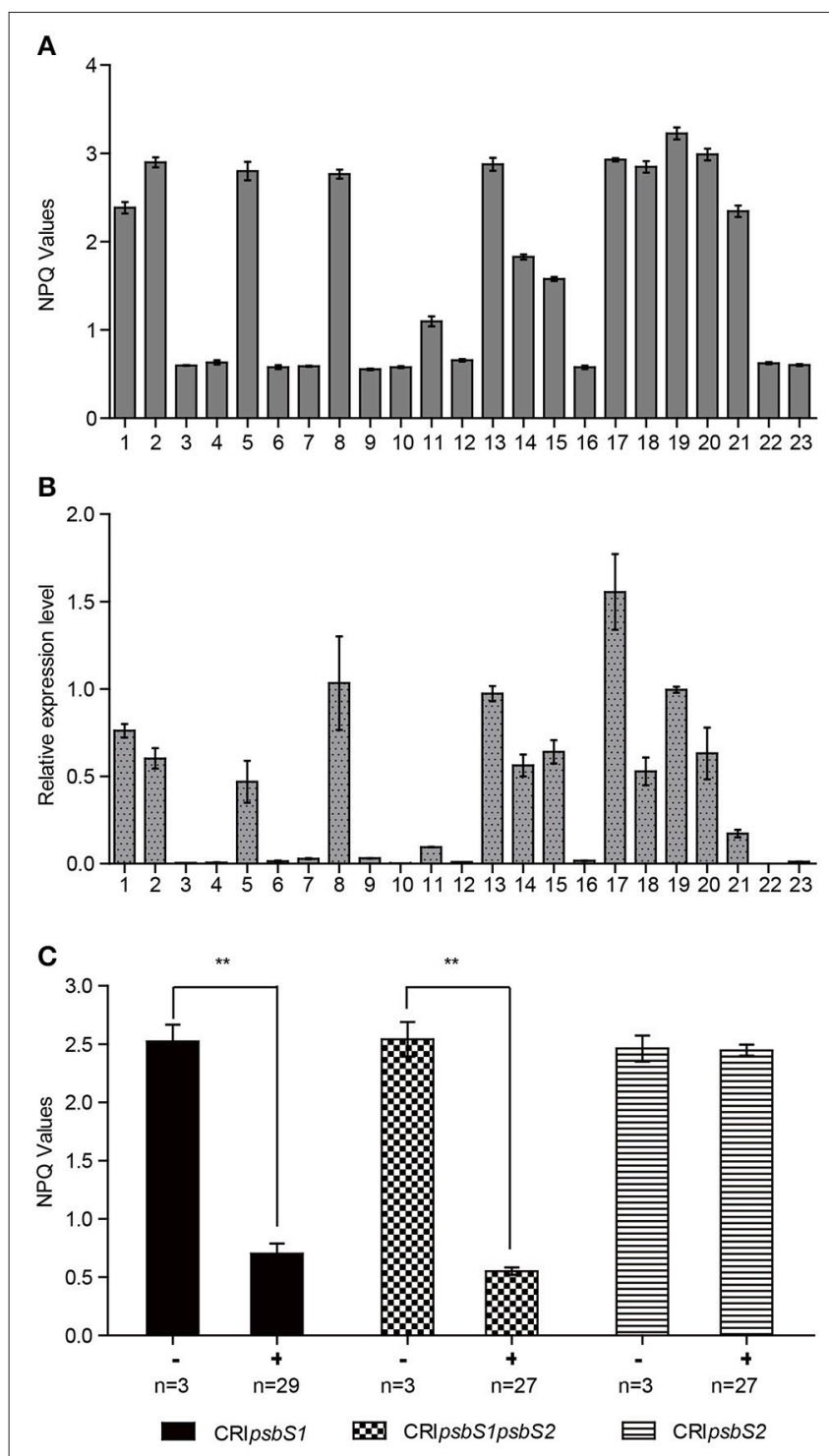

FIGURE 3 | The NPQ values in OsPsbS1 RNAi and CRISPR mutant plants. (A,B) The expression levels of OsPsbS1 (A) and corresponding NPQ values (B) in flag leaves of OsPsbS1 RNAi plants. (C) The NPQ values in CRISPR mutants targeting the single gene OsPsbS1 or OsPsbS2 and both of the two genes. The number of plants $(n)$ were shown. ${ }^{*}$ The differences in NPQ values between the wild type plants $(-)$ and mutants $(+)$ are significant at $P<0.01$.

initiation site), 5' UTR, 3' UTR, and non-synonymous SNPs and InDels in the coding region. We only focused on those haplotypes shared by at least 5 accessions in the analyzed group. Five haplotypes were found in the whole association panel (Figure S1). In total, five SNPs in the promoter region, three non-synonymous SNPs in the coding region, and seven SNPs in $3^{\prime}$ UTR were detected. Hap1, hap2, and hap3 were the three major haplotypes in indica group, whereas hap1 was the only major haplotype in japonica group (Figure S1). Considering the complexity of population structure and genetic background, we performed statistical analysis in the same or similar groups. Comparisons of the mean values of NPQ_13 showed that hap2 had significantly lower value than hap1 $\left(P=4.479 \times 10^{-12}\right)$ and hap3 $\left(P=1.227 \times 10^{-6}\right)$ in indica group (Figure 5). In addition, hap2 also exhibited significantly lower value than hap1 $(P=$ $0.0019)$ and hap3 $(P=0.0260)$ in NPQ_12 in indica. We checked the expression of LOC_Os01g01340 in 59 accessions randomly chosen from indica with different haplotypes. Using qRT-PCR analysis, it was found that expression levels of LOC_Os01g01340 in hap 1 accessions were much higher than those in hap2 and hap3 accessions $(P<0.01)$ (Figure 5, Table S5). These results indicate that this gene might be a good candidate for the GWAS locus.

\section{DISCUSSION}

The qE-type of NPQ is a very important photoprotective strategy for rice to adapt to the highly excessive natural illumination during summer growing season as well as the very dynamic light microenvironments inside canopy, which may be influenced minute by minute by wind, cloud, and time of day. In present study, using a diverse worldwide collection of 529 O. sativa accessions as the GWAS platform, we detected a total of 33 significant association loci for $\mathrm{qE}$ capacity. The validity of the GWAS signals was further demonstrated with three linkage mapping populations. All significant QTLs detected in biparental linkage mapping population could correspond to at least one GWAS signal. Compared with the intervals QTL mapping approach, our GWAS provided much higher resolution, facilitating candidate gene identification.

In this study, only three significant association regions were commonly detected by GWAS in two different groups. Although a total of 12,6 , and 4 significant association regions at LMM model were identified in indica, japonica, and aus subpopulations, respectively, there were no common QTLs detected between either of the two different subpopulations. These results indicate significant genetic heterogeneity of NPQ factors in different groups. We speculate that some of these QTLs might be fixed for one major haplotype in the subpopulation that failed in detection. For example, OsPsbS1 can only be detected in the whole population but not in each subpopulation, with the main reason lying in that the major haplotype (presence or absence of the 2,674-bp insertion in the promoter region) was fixed and few polymorphisms were present in each subpopulation. In addition, consistent with the previous report by Demming-Adams and Adams (1994), we also observed that NPQ values were sensitive to environmental changes. Although the phenotyping was conducted for two years on an identical set of 529 O. sativa accessions under very similar cultivation conditions, only two regions could be repeatedly detected by GWAS in both 2012 and 2013. NPQ values in 2013 were significant higher than that in 2012, which might be due to the hotter and less cloudy days during the 2013 summer season.

Our GWAS clearly reveals OsPsbS1 as a major determinant for natural variation of nonphotochemical quenching capacity in rice. OsPsbS1 was repeatedly detected and explained more than $40 \%$ of the variation in the whole association population in both 2012 and 2013, and demonstrated to be a common 


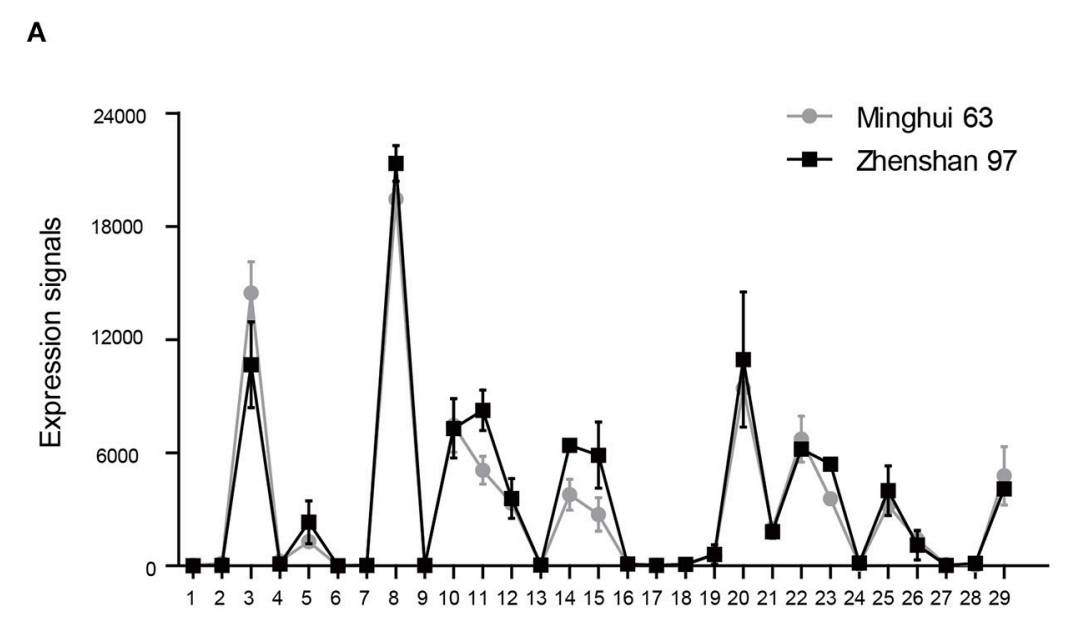

B
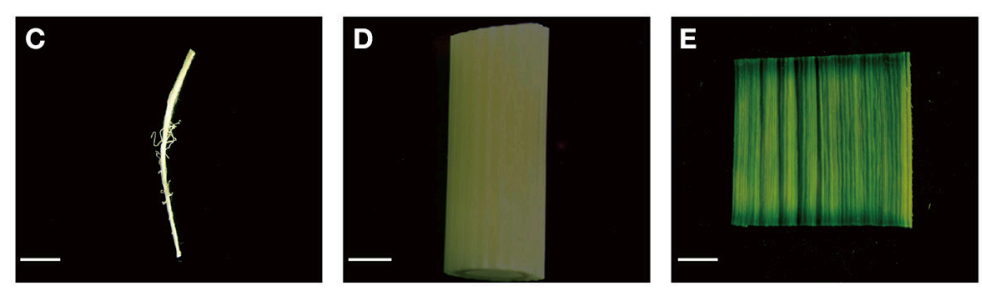

'
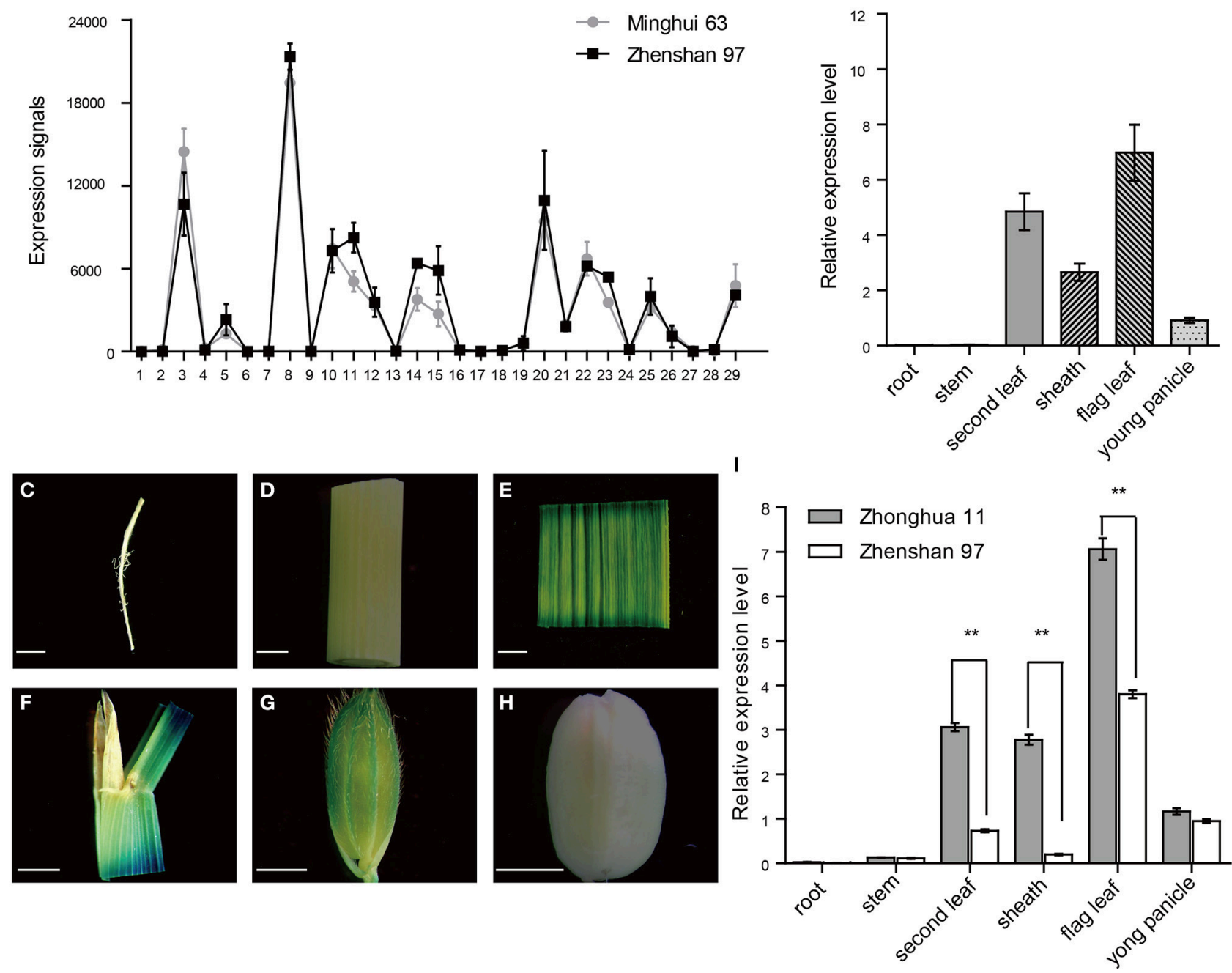

FIGURE 4 | Expression patterns of OsPsbS1. (A) Expression signals of OsPsbS1 in various tissues of Minghui 63 and Zhenshan 97 based on the microarray data. The X-axis represents the developmental stages which are listed in Table S1. The Y-axis represents the expression signals. (B) Real-time PCR analysis of OsPsbS1 in various tissues of 02428. (C-H) P OsPsbs1::GUS analysis in various tissues of Zhonghua 11. GUS staining shown in primary root (C), developing culm (D), flag leaves $\mathbf{( E )}$, leaf sheath $\mathbf{( F )}$, young glume $\mathbf{( G )}$, and mature seed $\mathbf{( H )}$. Scale bars $=2 \mathrm{~mm}$. (I) Comparison of the expression levels of OsPsbS1 between Zhonghua 11 and Zhenshan 97 with different genotypes in the OsPsbS1 promoter region by Real-time PCR. ${ }^{*}$ The differences in expression levels between Zhonghua 11 and Zhenshan 97 are significant at $P<0.01$.

major QTL in all three F2 mapping populations derived from inter-group crosses. It has been reported that, unlike typical light-harvesting complex proteins with three transmembrane helices, the PsbS protein has four helices that span the thylakoid membrane. Protein sequence analysis showed high similarity between helix I and helix III and also between helix II and helix IV. The $\mathrm{pH}$-sensing mechanism of the PsbS protein in Arabidopsis is influenced by two pairs of symmetrically arranged glutamate residues, Glu-122 and Glu-226, each located within or close to the two lumen-exposed loops of the protein ( $\mathrm{Li}$ et al., 2000, 2004). In this study, by sequencing 480 accessions, we found no nucleotide polymorphic sites for OsPsbS1 in exon-1, intron-1, and exon-2, and only one synonymous SNP in exon3 , indicating the structure and function of PsbS1 protein are highly conserved in rice. In Arabidopsis mutants lacking PsbS are specifically defective in $\mathrm{qE}$ and more sensitive to photoinhibition (Li et al., 2002), and show decreased fitness under fluctuating light conditions in the field (Külheim et al., 2002). Consistent with the reports in Arabidopsis, we also observed CRISPR mutants for OsPsbS1 exhibited drastically decreased NPQ values, and set very few seeds in the field. These findings suggest that the photoprotective processes conferred by $\mathrm{qE}$ serve fundamental roles in maintaining survival, reproduction, and fitness in plants. 
A

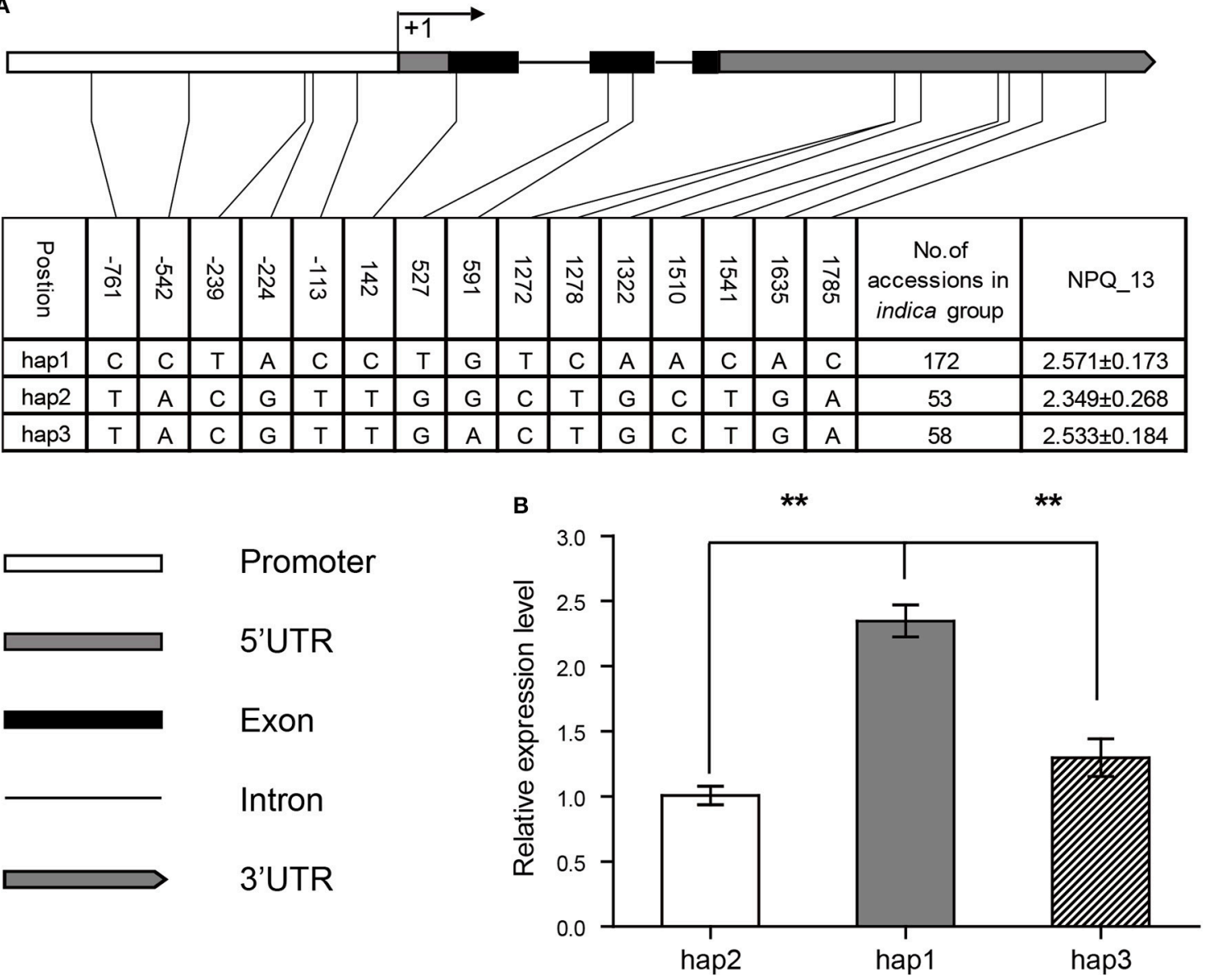

FIGURE 5 | Haplotypes (A) and expression levels (B) of the candidate gene (LOC_Os01g01340) in indica group. Three major haplotypes were detected, and the number of accessions in each haplotype is shown. The positions of SNPs are shown in the first row. Trait value (NPQ_13) is indicated in the right column. Graphical representations of the gene structure and expression levels of the gene in different haplotypes are shown below. ${ }^{* *}$ Indicates the differences of expression levels between hap1 and hap2 or hap3 are significant at $P<0.01$.

In rice, there is another $P s b S$ homologue, OsPsbS2. In present study, the whole genomic DNA sequences around OsPsbS2 from 63 accessions in the association panel were also sequenced and analyzed (Table S2). Three synonymous SNPs and two non-synonymous SNPs were found in the exon. The putative transmembrane regions and glutamate residues are also conserved in PsbS2. The expression patterns were very similar to OsPsbS1 (Figure S2). However, we didn't find significant function for OsPsbS2 in its CRISPR mutants under the same conditions as OsPsbS1 mutants, although there might be an additive effect in the double mutants when both OsPsbS1 and OsPsbS2 were knocked out (Figure 3C).

Due to the complexity of nature, identification of candidate genes for GWAS loci is generally difficult. The molecular mechanism underlying NPQ has been extensively investigated in Arabidopsis (Niyogi et al., 1998, 2001; Müller et al., 2001; Ruban, 2016). It is likely that the detected GWAS loci are rice homologues to the NPQ regulators identified in Arabidopsis. In addition to OsPsbS1 and OsPsbS2, we also compared positions of the significant GWAS signals with xanthophyll cycle related rice homologues which include OsVDE (LOC_Os04g31040) and OsZEP (LOC_Os04g37619), lutein synthesis related homologues which include OsLUT1A (LOC_Os10g39930), OsLUT1B (LOC_Os02g57290), OsLUT1C (LOC_Os02g07680), and OsLUT2 (LOC_Os01g39960), and cyclic electron flow related homologues which include OsPGR5 (LOC_Os08g45190), OsPGRL1A (LOC_Os08g41460), and OsPGRL1B (LOC_Os03g64020) (DalCorso et al., 2008; Kasajima et al., 2011). However, except OsPsbS1, none of these rice homologues were found to correspond to the detected GWAS loci. Close to the GWAS peak signals, we did find some candidate genes (Table 2) that might be involved in processes of electron transport, buildup of proton gradient, PSII light harvesting antenna structure and light harvesting complex II (LHCII) rearrangements that trigger and regulate NPQ scenario (Ruban, 2016). Considering the strong population structure and complicated genetic background, we further chose a candidate gene (LOC_Os01g01340) close to one representative GWAS 
peak signal for NPQ_13 in indica group and analyzed in depth. The results support that it might be a good candidate for the GWAS locus. Before our study, the function of this gene hasn't been reported. In addition, it is interesting to note that, Grain number, plant height, and heading date7 (Ghd7), a gene with pleiotropic effects on plant height, heading date, yield traits, and chlorophyll content (Xue et al., 2008; Wang et al., 2015), is in the very close proximity of one representative GWAS peak signal (around 9,162,584 on chromosome 7) that repeatedly detected in both NPQ_12 and NPQ_13. We thus suggest it as candidate gene for the GWAS locus (Table 2).

Manipulating photoprotective mechanism has been suggested and demonstrated to be an effective means to enhance both stress resistance and photosynthetic productivity of crop plants (Horton, 2000; Zhu et al., 2010; Murchie and Niyogi, 2011; Kromdijk et al., 2016). This study unraveled that OsPsbS1 is a major determinant for natural variation of $\mathrm{qE}$ capacity. In addition, we revealed dozens of significant association loci, most of which explained more than $5.0 \%$ of the phenotypic variance. In future, QTLs accelerating the recovery of NPQ should also be identified. Systematic combination and manipulation of these QTLs may increase qE capacity, enhance photoprotection, and improve rice photosynthesis and yield in adverse environments.

\section{CONCLUSIONS}

Thirty-three significant association loci for natural variation of NPQ capacity in rice were identified by GWAS, and the validity of the GWAS signals was demonstrated by linkage mapping in three F2 populations. Candidate genes underlying some significant association regions were proposed. OsPsbS1 was found to be a major determinant for NPQ variation explaining more than $40 \%$ of the variation in the whole association population, and a common major QTL in all mapping populations derived from inter-group crosses. The nucleotide diversity analysis reveals no non-synonymous SNPs or InDels in the coding region, indicating the PsbS1 protein sequence is highly conserved. Haplotypes with the 2,674-bp

\section{REFERENCES}

Bianchi, S. D., Ballottari, M., Dall'Osto, L., and Bassi, R. (2010). Regulation of plant light harvesting by thermal dissipation of excess energy. Biochem. Soc. Trans. 38, 651-660. doi: 10.1042/BST0380651

Bilger, W., and Björkman, O. (1990). Role of the xanthophyll cycle in photoprotection elucidated by measurements of light-induced absorbance changes, fluorescence and photosynthesis in leaves of Hedera canariensis. Photosynth. Res. 25, 173-185. doi: 10.1007/BF00033159

DalCorso, G., Pesaresi, P., Masiero, S., Aseeva, E., Schünemann, D., Finazzi, G., et al. (2008). A complex containing PGRL1 and PGR5 is involved in the switch between linear and cyclic electron flow in Arabidopsis. Cell 132, 273-285. doi: 10.1016/j.cell.2007.12.028

Demming-Adams, B., and Adams, W. W. (1994). Capacity for energy dissipation in the pigment bed in leaves with different xanthophyll cycle pools. Aust. J. Plant Physiol. 21, 575-588. doi: 10.1071/PP9940575

Du, H., Ouyang, Y., Zhang, C., and Zhang, Q. (2011). Complex evolution of S5, a major reproductive barrier regulator, in the cultivated rice Oryza sativa and its wild relatives. New Phytol. 191, 275-287. doi: 10.1111/j.1469-8137.2011.03691.x insertion in the promoter region exhibited significantly higher NPQ values and higher expression levels of OsPsbS1 in leaves. The OsPsbS1 RNAi plants and CRISPR mutants exhibited drastically decreased NPQ values. OsPsbS1 had specific and high-level expression in green tissues. A candidate gene encoding light-induced protein 1-like (LOC_Os01g01340) was also analyzed. The significant loci detected in present study would help enhance photoprotection and improve photosynthesis in rice.

\section{AUTHOR CONTRIBUTIONS}

GW designed the study and analyzed the data. QW carried out most of the experiments and analyzed the data. HZ and WX performed GWAS. Other authors assisted in experiments. GW and QW wrote the manuscript. JJ and JX performed genotypic assay in QTL mapping. XF and CL measured NPQ values. YH assisted in the construction of mapping populations.

\section{FUNDING}

This work was supported by grants from the Ministry of Agriculture of China (2016ZX08009001) and National 863 Project (No. 2014AA10A600).

\section{ACKNOWLEDGMENTS}

We thank Mr. H. X. Wang for his excellent work in field management. We appreciate Professor Yaoguang Liu (South China Agriculture University) kindly providing the CRISPR/Cas9 vector system for multiplex targeting of gene sites in monocot plants.

\section{SUPPLEMENTARY MATERIAL}

The Supplementary Material for this article can be found online at: https://www.frontiersin.org/articles/10.3389/fpls.2017. 01773/full\#supplementary-material

Horton, P. (2000). Prospects for crop improvement through the genetic manipulation of photosynthesis: morphological and biochemical aspects of light capture. J. Exp. Bot. 51, 475-485. doi: 10.1093/jexbot/51.suppl_1.475

Huang, X., Wei, X., Sang, T., Zhao, Q., Feng, Q., Zhao, Y., et al. (2010). Genomewide association studies of 14 agronomic traits in rice landraces. Nat. Genet. 42, 961-967. doi: 10.1038/ng.695

Jung, H., and Niyogi, K. K. (2009). Quantitative genetic analysis of thermal dissipation in Arabidopsis. Plant Physiol. 150, 977-986. doi: 10.1104/pp.109.137828

Kasajima, I., Ebana, K., Yamamoto, T., Takahara, K., Yano, M., KawaiYamada, M., et al. (2011). Molecular distinction in genetic regulation of nonphotochemical quenching in rice. Proc. Natl. Acad. Sci. U.S.A. 108, 13835-13840. doi: 10.1073/pnas.1104809108

Kromdijk, J., Glowacka, K., Leonelli, L., Gabilly, S. T., Iwai, M., Niyogi, K. K., et al. (2016). Improving photosynthesis and crop productivity by accelerating recovery from photoprotection. Science 354, 857-861. doi: 10.1126/science.aai8878

Külheim, C., Ågren, J., and Jansson, S. (2002). Rapid regulation of light harvesting and plant fitness in the field. Science 297, 91-93. doi: 10.1126/science.1072359 
Li, M., Yeung, J., Cherny, S., and Sham, P. (2012). Evaluating the effective numbers of independent tests and significant $\mathrm{p}$-value thresholds in commercial genotyping arrays and public imputation reference datasets. Hum. Genet. 131, 747-756. doi: 10.1007/s00439-011-1118-2

Li, X., Björkman, O., Shih, C., Grossman, A. R., Rosenquist, M., Jansson, S., et al. (2000). A pigment-binding protein essential for regulation of photosynthetic light harvesting. Nature 403, 391-395. doi: 10.1038/35000131

Li, X., Gilmore, A. M., Caffarri, S., Bassi, R., Golan, T., Kramer, D., et al. (2004). Regulation of photosynthetic light harvesting involves intrathylakoid lumen $\mathrm{pH}$ sensing by the PsbS protein. J. Biol. Chem. 279, 22866-22874. doi: 10.1074/jbc.M402461200

Li, X., Müller-Moulé, P., Gilmore, A. M., and Niyogi, K. K. (2002). PsbSdependent enhancement of feedback de-excitation protects photosystem II from photoinhibition. Proc. Natl. Acad. Sci. U.S.A. 99, 15222-15227. doi: 10.1073/pnas.232447699

Li, Z., Wakao, S., Fischer, B. B., and Niyogi, K. K. (2009). Sensing and responding to excess light. Annu. Rev. Plant Biol. 60, 239-260. doi: 10.1146/annurev.arplant.58.032806.103844

Lippert, C., Listgarten, J., Liu, Y., Kadie, C. M., Davidson, R. I., and Heckerman, D. (2011). FaST linear mixed models for genome-wide association studies. Nat. Methods 8, 833-835. doi: 10.1038/nmeth.1681

Müller, P., Li, X., and Niyogi, K. K. (2001). Non-photochemical quenching. a response to excess light energy. Plant Physiol. 125, 1558-1566. doi: $10.1104 /$ pp.125.4.1558

Murchie, E. H., and Niyogi, K. K. (2011). Manipulation of photoprotection to improve plant photosynthesis. Plant Physiol. 155, 86-92. doi: $10.1104 /$ pp. 110.168831

Nilkens, M., Kress, E., Lambrev, P., Miloslavina, Y., Müller, M., Holzwarth, A. R., et al. (2010). Identification of a slowly inducible zeaxanthin-dependent component of non-photochemical quenching of chlorophyll fluorescence generated under steady-state conditions in Arabidopsis. Biochim. Biophys. Acta. 1797, 466-475. doi: 10.1016/j.bbabio.2010.01.001

Niyogi, K. K., Grossman, A. R., and Björkman, O. (1998). Arabidopsis mutants define a central role for the xanthophyll cycle in the regulation of photosynthetic energy conversion. Plant Cell 10, 1121-1134. doi: $10.1105 /$ tpc.10.7.1121

Niyogi, K. K., Shih, C., Chow, W. S., Pogson, B. J., Dellapenna, D., and Björkman, O. (2001). Photoprotection in a zeaxanthin- and lutein-deficient double mutant of Arabidopsis. Photosynth. Res. 67, 139-145. doi: 10.1023/A:1010661102365
Nuruzzaman, M., Kanno, T., Amada, R., Habu, Y., Kasajima, I., Ishikawa, T., et al. (2014). Does the upstream region possessing MULE-like sequence in rice upregulate PsbS1 gene expression? PLoS ONE 9:e102742. doi: 10.1371/journal.pone.0102742

Ruban, A. V. (2016). Nonphotochemical chlorophyll fluorescence quenching: mechanism and effectiveness in protecting plants from photodamage. Plant Physiol. 170, 1903-1916. doi: 10.1104/pp.15.01935

Wang, L., Xie, W., Chen, Y., Tang, W., Yang, J., Ye, R., et al. (2010). A dynamic gene expression atlas covering the entire life cycle of rice. Plant J. 61, 752-766. doi: 10.1111/j.1365-313X.2009.04100.x

Wang, Q., Xie, W., Xing, H., Yan, J., Meng, X., Li, X., et al. (2015). Genetic architecture of natural variation in rice chlorophyll content revealed by genome wide association study. Mol. Plant 8, 946-957. doi: 10.1016/j.molp.2015.02.014

Xie, W., Wang, G., Yuan, M., Yao, W., Lyu, K., Zhao, H., et al. (2015). Breeding signatures of rice improvement revealed by a genomic variation map from a large germplasm collection. Proc. Natl. Acad. Sci. U.S.A. 112, E5411-E5419. doi: $10.1073 /$ pnas. 1515919112

Xue, W., Xing, Y., Weng, X., Zhao, Y., Tang, W., Wang, L., et al. (2008). Natural variation in Ghd7 is an important regulator of heading date and yield potential in rice. Nat Genet. 40, 761-767. doi: 10.1038/ng.143

Zhao, K., Tung, C. W., Eizenga, G. C., Wright, M. H., Ali, M. L., Price, A. H., et al. (2011). Genome-wide association mapping reveals a rich genetic architecture of complex traits in Oryza sativa. Nat. Commun. 2:467. doi: 10.1038/ncomm s1467

Zhu, X., Long, S., and Ort, D. (2010). Improving photosynthetic efficiency for greater yield. Annu. Rev. Plant Biol. 61, 235-261. doi: 10.1146/annurev-arplant-042809-112206

Conflict of Interest Statement: The authors declare that the research was conducted in the absence of any commercial or financial relationships that could be construed as a potential conflict of interest.

Copyright (c) 2017 Wang, Zhao, Jiang, Xu, Xie, Fu, Liu, He and Wang. This is an open-access article distributed under the terms of the Creative Commons Attribution License (CC BY). The use, distribution or reproduction in other forums is permitted, provided the original author(s) or licensor are credited and that the original publication in this journal is cited, in accordance with accepted academic practice. No use, distribution or reproduction is permitted which does not comply with these terms. 